\title{
Neurology patients online: perceptions and reality
}

\author{
John W Henson
}

There is a perception among neurologists that most patients actively use the internet to access information about their illness, raising concerns about the potential for misinformation, and the possibility of patients using precious minutes during clinic visits for discussion of internetderived information of limited relevance to their condition. A new study from the University of Turin (Chiò A et al. [2008] Eur J Neurol 15: 55-60) sheds critical light on the informationseeking behavior of patients in an era of exploding online health information. By understanding this behavior, neurologists can help patients in their search for information.

Chiò et al. interviewed 60 patients with amyotrophic lateral sclerosis (ALS) and their caregivers regarding use of the internet as a source of medical information. Several interesting features emerged from the data. Twothirds of the patients felt that their physicians provided all relevant clinical information about ALS in the clinical setting, and $55 \%$ of patients and $83 \%$ of caregivers expressed the need to learn more about the disease outside the clinical setting. The internet was the most common source for information outside the clinic, but, surprisingly, only $37 \%$ of patients and $63 \%$ of caregivers actually derived information from online sources. These numbers are comparable to those found in two older studies (Diaz JA et al. [2002] J Gen Intern Med 17: 180-185; Baker L et al. [2003] JAMA 289: 2400-2406), indicating that the fraction of patients using the internet for health information has remained relatively stable over the past few years. Major media outlets, including newspapers and television, were the second most common sources of medical information for patients with ALS.

The top three internet search goals were disease outcome, disease-modifying therapies, and scientific research. Caregivers were more interested in finding experimental treatments and alternative therapies than were the patients. Interestingly, patients were ambivalent
The top three

internet

search goals

were disease

outcome,

disease-

modifying

therapies, and scientific

research

JW Henson is Associate Editor for Science for AAN. com, the American Academy of Neurology, a Neurologist and Radiologist at Massachusetts General Hospital, and Associate Professor of Neurology, Harvard Medical School, Boston, MA, USA.

\section{Competing interests}

The author declared no competing interests.

www.nature.com/clinicalpractice doi:10.1038/ncpneuro0852 about the reliability of the information gained from the internet: information from medical meetings, television and patient associations was deemed more trustworthy.

Age and sex did not correlate with the likelihood of pursuing online health information. Other studies have shown higher rates of health-related use of the internet with increasing educational level; data on age, sex and income have provided conflicting results.

What recommendations can neurologists give to their patients regarding use of the internet to find medical information? The website of the National Institutes of Neurological Disorders and Stroke (NINDS) offers an excellent patientoriented resource. The NINDS 'Disorders Index' (http://www.ninds.nih.gov/disorders/disorder_ index.htm) provides moderately detailed information in nontechnical language about a large number of neurological conditions and diseases. Links are provided to the websites of national, disease-specific, not-for-profit organizations from which patients can obtain additional information and assistance. For those patients who want to learn about the latest clinical trial related to their condition, there is also a link to a preselected list of trials that are registered with ClinicalTrials.gov. On the ALS page, 44 studies were listed as actively recruiting patients at the time of writing. The list of trials can be refined by characteristics such as subject age, location of study by state, and study type. This system allows both neurologists and their patients to search for appropriate clinical trials. Patients can access a PubMed search for relevant medical literature on any disorder. Finally, a link to 'Additional Resources from Medline Plus' leads to a large amount of additional, patient-oriented information provided by the US National Library of Medicine. These are all outstanding resources that meet the top three internet search goals defined in the Chiò et al. study, and to which neurologists can safely direct their patients. 